\title{
CONTINUOUS MEASURES AND LACUNARITY ON HYPERGROUPS
}

\author{
BY
}

RICHARD C. VREM

\begin{abstract}
The relationship between measures on a compact hypergroup $K$ whose Fourier-Stieltjes transforms vanish at infinity and the space $M_{c}(K)$ of continuous measures is studied. Examples are provided of measures $\mu$ with $\hat{\mu}$ vanishing at infinity and $\mu \in M_{c}(K)$. Sufficient conditions are given for $\hat{\mu} \in c_{0}(\hat{K})$ to imply $\mu \in M_{c}(K)$. An investigation of Helson sets on compact abelian hypergroups is initiated and the study of Sidon sets on compact abelian hypergroups is continued. A class of compact abelian hypergroups is shown to have no infinite Helson sets and no infinite Sidon sets. This result generalizes results of D. L. Ragozin and D. Rider on central Sidon sets for compact connected Lie groups.
\end{abstract}

1. Introduction. The question of existence in a compact group of infinite central lacunary sets has been studied by J. F. Price [7], D. L. Ragozin [8], D. Rider [9] and A. H. Dooley [2, 3], among others. In this paper, the framework of compact hypergroups (see R. Vrem [13] for the connection between hypergroups and central lacunarity on compact groups) is used to study this question in more generality.

In $\$ 2$ the relationship between continuous measures and those measures whose Fourier-Stieltjes transforms vanish at infinity is investigated. Sufficient conditions are given in order for a measure's transform vanishing at infinity to imply the measure is continuous as well as examples to show this implication need not always hold. Sidonicity on compact abelian hypergroups is the subject of §3. A sufficient condition for a hypergroup to contain an infinite Sidon set is given and a result of Ross [10] is used to determine a class of hypergroups which have no infinite Sidon sets. $\$ 4$ initiates a study of Helson sets on compact abelian hypergroups. The standard equivalent properties of Helson sets are established. It is shown that no infinite subhypergroup can be a Helson set and that no nonzero measure with infinite support can be supported on a Helson set. Finally, in $\$ 5$ examples are considered. In particular, results of D. L. Ragozin [8] and D. Rider [9] on central Sidon sets for compact connected semisimple Lie groups are generalized as well as a result of C. F. Dunkl and D. E. Ramirez [4] on Helson sets.

The reader is referred to $R$. Vrem $[13,14]$ or $R$. Jewett [6] for any unexplained notation. $M(K)$ will denote the space of all finite regular Borel measures on the hypergroup $K, \delta_{x}$ is the point mass at $x, x \mapsto x^{v}$ is the involution on $K$ and $I_{A}$ denotes the indicator function of $A$.

Received by the editors January 5, 1981 and, in revised form, March 5, 1981.

1980 Mathematics Subject Classification. Primary 43A05, 43A46; Secondary 43A75. 
2. Continuous measures. Throughout this section we assume $K$ is a compact (not necessarily abelian) hypergroup with dual object $\hat{K}$ and normalized Haar measure $m$. A measure $\mu \in M(K)$ is continuous if $\mu(\{x\})=0$ for all $x \in K$. We denote the set of all continuous Borel measures on $K$ by $M_{c}(K)$. We begin with a lemma (compare with [5, 34.23]).

LEMMA 2.1. Let $K$ be a compact hypergroup with $V$ any neighborhood of $e$ and $V_{1} a$ symmetric neighborhood of e such that $V_{1} * V_{1} \subseteq V$. Let $F$ be a compact set such that $F \subseteq V_{1}$ and $m(F)>\frac{1}{4} m\left(V_{1}\right)$. If $g=m(F)^{-1} I_{V_{1}} * I_{F}$ then

(i) $g \in A(K)$ (the space of all continuous functions on $K$ with absolutely convergent Fourier series);

(ii) $g(x)=1$ for all $x$ is some neighborhood of $e$;

(iii) $g(x)=0$ for all $x \in K-V$;

(iv) $\|\hat{g}\|_{1}<2$.

Proof. Since $g$ is the convolution of two $L^{2}(K)$ functions it is in $A(K)$ by [14, 4.9]. Using [6, 5.5 and 7.2] we have

$$
g(x)=m(F)^{-1} \int_{K} I_{F}(y) I_{V_{1}}\left(x * y^{v}\right) d m(y) .
$$

There exists a symmetric neighborhood $V_{2}$ of $e$ such that $V_{2} * F_{2}^{v} \subseteq V_{1}$. The last containment and (1) show that $g(x)=1$ for all $x \in V_{2}$. Clearly, $g$ vanishes off $V_{1} * F \subseteq V$ which establishes (iii). Using [14, 4.8] it follows that

$$
\|\hat{g}\|_{1} \leqslant m(F)^{-1}\left\|I_{V_{1}}\right\|_{2}\left\|I_{F}\right\|_{2}<2 .
$$

The next lemma gives a technical condition satisfied by measures whose Fourier-Stieltjes transforms vanish at infinity.

LEMMA 2.2. Let $K$ be a compact hypergroup and suppose $\mu \in M(K)$ with $\hat{\mu} \in$ $\mathcal{E}_{0}(\hat{K})$. Let $\mathcal{Q}$ be an open basis of neighborhoods of e directed by reverse set inclusion. For each $V \in \mathcal{Q}$ select $a g_{V}$ as in Lemma 2.1. Then using the inner product of [5, 28.28] for $\mu \in M(K)$ we have

$$
\lim _{V}\left\langle\hat{\mu},\left(g_{V} * \mu\right)^{\wedge}\right\rangle=\sum_{x \in K}|\mu(\{x\})|^{2} \delta_{x} * \delta_{x^{v}}(\{e\}) .
$$

Proof. If $\mu \in M(K)$ then the inequality $\left\|\hat{g}_{V} \hat{\mu}\right\|_{1} \leqslant\left\|\hat{g}_{V}\right\|_{1}\|\hat{\mu}\|_{\infty}$ shows that $g_{V} * \mu \in A(K)$. It follows from $[12,4.25]$ that

$$
\left\langle\hat{\mu},\left(g_{V} * \mu\right)^{\wedge}\right\rangle=\int_{K} \overline{g_{V} * \mu} d \mu=\int_{K} g_{V} d \mu^{*} * \mu .
$$

The regularity of $\mu * \mu$ gives

$$
\lim _{V} \int_{K} g_{V} d \mu * \mu^{*}=\mu * \mu^{*}(\{e\})=\int_{K} \int_{K} \delta_{x} * \delta_{y}(\{e\}) d \mu(x) d \mu^{*}(y) .
$$


However, $e \in \operatorname{supp} \delta_{x} * \delta_{y}$ if and only if $y=x^{v}$ so we have

$$
\begin{aligned}
\mu * \mu^{*}(\{e\}) & =\int_{K} \delta_{x} * \delta_{x^{v}}(\{e\}) \overline{\mu(\{x\})} d \mu(x) \\
& =\sum_{x \in K} \delta_{x} * \delta_{x^{v}}(\{e\})|\mu(\{x\})|^{2} .
\end{aligned}
$$

Combining equations (2) and (3) we have the desired equality.

The following theorem generalizes [5, 34.45(b)].

THEOREM 2.3. Let $K$ be a compact hypergroup with the property that $\delta_{x} * \delta_{x^{\circ}}(\{e\})$ $>0$ for all $x \in K$. If $\mu \in M(K)$ with $\hat{\mu} \in \mathcal{E}_{0}(\hat{K})$ then $\mu$ is a continuous measure.

Proof. The proof that $\lim _{V}\left\langle\hat{\mu},\left(g_{V} * \mu\right)^{\wedge}\right\rangle=0$ follows from Lemma 2.1 and an argument similar to that found in $[5,34.45(\mathrm{~b})]$. Lemma 2.2 together with the condition that $\delta_{x} * \delta_{x}(\{e\})>0$ establish the theorem.

Some hypothesis like $\delta_{x} * \delta_{x}(\{e\})>0$ for all $x \in K$ is needed in order for Theorem 2.3 to hold. For example, let $K$ be an infinite compact hypergroup with an isolated point $x$ (see Example 2.6 below). If $m$ is normalized Haar measure on $K$ it is immediate that $\hat{m} \in \mathcal{E}_{0}(\hat{K})$ (use orthogonality relations $[14,2.6]$ ) but $m(\{x\})$ $\neq 0$. Further examples of measures which are not continuous but whose transforms are in $c_{0}(\hat{K})$ can be found among the abelian hypergroups which are $n$-fold absolutely continuous (see [10, 6.12-6.14 and 6.17]).

EXAMPLE 2.4. If $K$ is an abelian hypergroup which is $n$-fold absolutely continuous (i.e., $\delta_{x_{1}} * \cdots * \delta_{x_{n}} \in M_{a}(K)$ for all $x_{i} \in K-Z$ ) then the $n$-fold product of $x$ with itself $\delta_{x} * \cdots * \delta_{x}$ satisfies $\left(\delta_{x} * \cdots * \delta_{x}\right)^{\wedge} \in c_{0}(\hat{K})$ and hence $\left(\delta_{x}\right)^{\wedge} \in c_{0}(\hat{K})$, i.e. each point mass of $K-Z$ has a transform in $c_{0}(\hat{K})$.

The next theorem illustrates that the phenomenon found in Example 2.4 is not atypical.

THEOREM 2.5. Suppose $K$ is a compact abelian hypergroup. There exists a measure $\mu \in M(K)-M_{c}(K)$ with $\hat{\mu} \in c_{0}(\hat{K})$ if and only if there is an $x \in K$ with $\left(\delta_{x}\right)^{\wedge} \in c_{0}(\hat{K})$.

Proof. Sufficiency is clear. If $\mu \in M(K)-M_{c}(K)$ with $\hat{\mu} \in c_{0}(\hat{K})$ then there is an $x \in K$ with $\mu(\{x\}) \neq 0$. If $\varepsilon>0$ is any positive number then since $\hat{\mu} \in c_{0}(\hat{K})$ there is a finite subset $S \subseteq \hat{K}$ with $|\hat{\mu}(\chi)| \leqslant \varepsilon|\mu(\{x\})|$ for all $\chi \in K^{\wedge}-S$. It follows that

$$
\begin{aligned}
\varepsilon^{2}|\mu(\{x\})|^{2} & \geqslant|\hat{\mu}(\chi)|^{2}=\int_{K} \bar{\chi} d \mu * \mu^{*}=\int_{K} \int_{K} \bar{\chi}(s) \bar{\chi}(t) d \mu(s) d \mu^{*}(t) \\
& =|\chi(x)|^{2}|\mu(\{x\})|^{2}+c \text { (a positive number) }
\end{aligned}
$$

and hence $|\chi(x)| \leqslant \varepsilon$ for all $\chi \in \hat{K}-S$. This shows that $\left(\delta_{x}\right)^{\wedge} \in c_{0}(\hat{K})$.

Other pathologies relating to $M_{c}(K)$ can occur for compact abelian hypergroups. It is easy to see that $M_{c}(K)$ is always a closed subspace of $M(K)$ for any locally compact hypergroup $K$ and if $K$ is a group then $M_{c}(K)$ is an ideal in $M(K)$. However, the next example shows that $M_{c}(K)$ need not be an ideal in $M(K)$ if $K$ is a hypergroup. 
EXAMPLE 2.6. Let $H=\{x, e\}$ be the two-point hypergroup with convolution given by $\delta_{x} * \delta_{x}=\frac{1}{2} \delta_{e}+\frac{1}{2} \delta_{x}$. Using $[6,10.5]$ we form the join of $H$ and $G$ (any nondiscrete compact abelian group). Now $K=H \vee G$ is clearly a compact abelian hypergroup. Let $\mu$ be any nonzero continuous measure supported on $K-\{x\}=G$. Next we consider $\delta_{x} * \mu$ and show that it is not continuous. Using the fact that $\delta_{x} * \delta_{t}=\delta_{x}$ for all $t \in K-\{x\}$ we have

$$
\begin{aligned}
\delta_{x} * \mu(\{x\}) & =\int_{K} I_{\{x\}}(x * t) d \mu(t)=\int_{K-\{x\}} I_{\{x\}}(x * t) d \mu(t) \\
& =\mu(K-\{x\})>0,
\end{aligned}
$$

i.e. $\delta_{x} * \mu \notin M_{c}(K)$ and hence $M_{c}(K)$ is not an ideal in $M(K)$.

3. Sidon sets. In this section the question of which compact abelian hypergroups admit infinite Sidon sets is discussed. For general results regarding Sidon sets on compact hypergroups the reader is referred to [13]. The first theorem gives a sufficient condition for a compact abelian hypergroup to admit an infinite Sidon set.

THEOREM 3.1. If $K$ is a compact abelian hypergroup with an infinite maximal subgroup $Z$, then $K$ admits an infinite Sidon set.

Proof. Let $\lambda: \hat{K} \rightarrow \hat{Z}$ be the restriction map. By $[10,3.6] \lambda$ is a continuous map of $\hat{K}$ onto $\hat{Z}$. Since $Z$ is an infinite compact abelian group it has an infinite Sidon set, say $S \subseteq \hat{Z}$. Let $Z_{\chi}=\{\phi \in \hat{K}: \lambda(\phi)=\chi\}$ for $\chi \in \hat{Z}$ and consider $\tilde{S} \subseteq \hat{K}$ found by selecting an element of $Z_{\chi}$ for each $\chi \in S$. For each $\Phi \in l_{\infty}(S)$ there is a $\mu \in M(Z)$ such that $\hat{\mu}=\Phi$. Extending $\mu$ to $\tilde{\mu}$ on $K$ by setting $\tilde{\mu}=0$ on $K-Z$ we clearly have $\hat{\tilde{\mu}}=\hat{\mu}=\Phi$. Thus $\tilde{S}$ is Sidon by $[13,2.2]$.

The next theorem uses the $n$-fold absolutely continuous hypergroups introduced by Ross in [10] to give a class of hypergroups which admit no infinite Sidon sets.

THEOREM 3.2. If $K$ is a compact abelian hypergroup which is $n$-fold absolutely continuous, with $Z$ finite and $\hat{K}$ a hypergroup, then $K$ admits no infinite Sidon sets.

Proof. First we show that we may assume $m(Z)=0$. For if not, there exists $x \in Z$ with $m(\{x\})>0$. Since $x \in Z$ (a group) it is easy to see that this would force $m(\{e\})>0$. [6, 7.1B] would then show that $K$ is finite and hence admits no infinite Sidon sets.

Thus we may assume $m(Z)=0$ so that the conditions of $[10,6.7]$ are satisfied. If $S$ is any infinite subset of $\hat{K}$ then $c_{0}(S)$ has infinite codimension in $l_{\infty}(S)$. Let $M_{h}(K)=\{\mu \in M(K):|\mu|(Z)=0\}$ and use [10, 6.7] to conclude $\left(M_{h}(K)\right)^{\wedge}=$ $c_{0}(\hat{K})$. But $M(K)=M(Z) \oplus M_{h}(K)$ by $[10,6.4]$ and $Z$ is finite so that $M_{S}(K)^{\wedge}=$ $c_{0}(S)+$ f.d. space. Hence $M_{S}(K)^{\wedge} \neq l_{\infty}(S)$ which implies $S$ is not a Sidon set.

For examples of this phenomenon see Examples 5.1 and 5.2.

4. Helson sets. Let $K$ be a compact abelian hypergroup with $A(K)$ the space of $L^{1}(K)$ functions with absolutely convergent Fourier series. We call a closed subset $F$ of $K$ a Helson set if $A(F)=C(F)$. The following theorem establishes a number of conditions which are equivalent to being a Helson set (compare with $[5,41.12]$ ). 
TheOREM 4,1. The following are equivalent for a closed subset $F$ of $K$,

(i) $F$ is a Helson set;

(ii) the set $\{\hat{\mu}: \mu \in M(F)\}$ is a closed subspace of $l_{\infty}(\hat{K})$;

(iii) there is a constant $c>0$ such that $\|\mu\| \leqslant c\|\hat{\mu}\|_{\infty}$ for all $\mu \in M(F)$;

(iv) there is a constant $c>0$ such that $\|g\|_{A(F)} \leqslant c\|g\|_{C(F)}$ for all $g \in A(F)$.

Moreover, (iii) holds with a given value of $c$ if and only if (iv) holds with the same value of $c$. In fact, $c$ is usually denoted by $\alpha(F)$ and called the Helson constant of $F$.

PROof. The proof will follow easily from the general Banach space result [5, E.9] with $E=l_{1}(\hat{K}), E^{*}=l_{\infty}(\hat{K}), E^{\prime}=C(F), E^{\prime *}=M(F)$ and $T$ the inverse Fourier transform once we establish that $T^{*}$ is injective. If $\mu \in M(F)$ then $T^{*} \mu=\mu \circ T$, i.e.

$$
\int_{F} f d \mu=\sum_{\chi \in \hat{K}} \hat{f}(\chi)\left(T^{*} \mu\right)(\chi)
$$

for all $f \in A(F)$. Applying $[6,12.2 \mathrm{C}]$ along with Fubini yields

$$
\int_{F} f d \mu=\int_{F} \sum \hat{f}(\chi) \chi(x) d \mu(\bar{x})=\sum_{\chi \in \hat{K}} \hat{f}(\chi) \hat{\mu}(\bar{\chi}) .
$$

Using equations (1) and (2) along with the uniqueness of the Fourier transform it follows that $T^{*}$ is injective.

The next theorem shows that no infinite subhypergroup of a compact abelian hypergroup can be a Helson set.

THEOREM 4.2. Suppose $K_{0}$ is a subhypergroup of the compact abelian hypergroup $K$. If $K_{0}$ is a Helson set then it is finite.

Proof. Since $K_{0}$ is a Helson set it follows that $A_{K_{0}}=C\left(K_{0}\right)$. Now $A\left(K_{0}\right) \subseteq$ $C\left(K_{0}\right)=A_{K_{0}}(K) \subseteq A\left(K_{0}\right)$ so that $A\left(K_{0}\right)=C\left(K_{0}\right)$ and hence $K_{0}$ is finite by [13, 2.11].

The next result shows that a measure whose Fourier-Stieltjes transform vanishes at infinity cannot be supported on a Helson set (compare with [5, 41.18] and [11, 5.6.10]).

THEOREM 4.3. Suppose that $F$ is a Helson set in the compact abelian, nondiscrete hypergroup $K$ with $\hat{K}$ also a hypergroup. If $\mu \in M(F)$ and $\operatorname{supp}(\mu)$ is infinite then $\hat{\mu} \notin c_{0}(\hat{K})$.

Proof. Let $M_{0}$ be the set of all $\mu \in M(K)$ such that $\hat{\mu} \in c_{0}(\hat{K})$. By Theorem 4.1, the mapping $\mu \mapsto \hat{\mu}$ carries $M_{0}$ onto a closed linear subspace of $c_{0}(\hat{K})$ and the inverse of the mapping $\mu \mapsto \hat{\mu}$ is also continuous. A standard application of the Hahn-Banach theorem shows that if $L$ is a bounded linear functional on $M_{0}$, then there is a $\phi \in L^{1}(K)$ such that

$$
L(\mu)=\sum_{\chi \in \hat{K}} \hat{\mu}(\chi) \phi(\chi)
$$


for all $\mu \in M_{0}$. By $[6,12.1]$

$$
\sum_{\chi \in \hat{K}} \hat{\mu}(\chi) \phi(\chi)=\int_{K} f(x) d \mu(x),
$$

where $f \in C(F)$ and $\hat{f}^{*}=\phi\left(f^{*}(x)=f\left(x^{v}\right)\right)$. Thus $L(\mu)=\int_{F} f d \mu$ for all $\mu \in M_{0}$. If $g \in B^{\infty}(F)$ then there is an $f \in C(F)$ such that

$$
\int_{F} g d \mu=\int_{F} f d \mu
$$

for all $\mu \in M_{0}$. An easy computation with $\bar{\chi} \mu(\chi \in \hat{K})$ and the uniqueness of the Fourier-Stieltjes transform shows that

$$
g \mu=f \mu
$$

for all $\mu \in M_{0}$.

Now we assume $M_{0}$ contains a nonzero measure $\mu \in M(F)$. First we show that we may assume that $\mu$ is a nonnegative measure. If not, we consider $|\mu|=g \mu$ where $g$ is selected as in [5, 14.12]. From equation (1) there is a function $f \in C(F)=A(F)$ such that $g \mu=f \mu$. For each $\chi \in \hat{K}$ we compute

$$
\begin{aligned}
|\mu|^{\wedge}(\chi) & =\int_{K} \bar{\chi}(t) f(t) d \mu(t)=\int_{K} \bar{\chi}(t) \sum_{\Psi \in \hat{K}} \hat{f}(\Psi) \Psi(t) \pi(\Psi) d \mu(t) \\
& =\sum_{\Psi \in \hat{K}} \hat{f}(\Psi) \hat{\mu}(\chi \bar{\Psi}) \pi(\psi)=\hat{f} * \hat{\mu}(\chi),
\end{aligned}
$$

where $\pi$ denotes Haar measure on $\hat{K}$ and the last convolution is over $\hat{K}$. Since $\hat{\mu} \in c_{0}(\hat{K}),[6,4.2 \mathrm{E}]$ implies $|\mu|^{\wedge} \in c_{0}(\hat{K})$ and hence $|\mu| \in M_{0}$.

Thus we may assume $\mu$ is nonnegative and since $\hat{\mu} \in c_{0}(\hat{K})$, for each $\varepsilon>0$, $\{\chi \in \hat{K}:|\hat{\mu}(\chi)|>\varepsilon\}$ is finite. Hence $A=\{\chi \in \hat{K}:|\hat{\mu}(\chi)| \neq 0\}$ is a countable subset of $\hat{K}$. Then $D=A \cup \bar{A}$ (where $\bar{A}=\{\bar{\chi}: \chi \in A\}$ ) is a countable subset of $\hat{K}$ which contains 1 . But $\hat{K}$ is discrete so $P=\cup_{n=1}^{\infty} D^{n}$ (where $D^{n}=$ $D * D * \cdots * D, n$-times) is a countable subhypergroup of $\hat{K}$.

In the notation of $[10,3.2], P^{\perp}=\{y \in K: \chi(y)=1$ for all $\chi \in P\}$ is a compact subhypergroup of $K$, which we denote by $K_{0}$. Let $\lambda_{0}$ be normalized Haar measure on $K_{0}$. Clearly, $\hat{\lambda}_{0} \hat{\mu}=\hat{\mu}$ so that $\mu=\lambda_{0} * \mu$. If $y \in K_{0}$ then $\delta_{y} * \mu=\delta_{y} * \lambda_{0} * \mu=\mu$ and hence by [6,3.2G], $S=\operatorname{supp}\left(\delta_{y} * \mu\right)=\{y\} * S$. This gives $K_{0} * S=S$ which implies $S$ is a union of cosets of $K_{0}$. Suppose $f \in C\left(K_{0}\right)$ and $y \in S$. Now supp $f_{y} \subseteq S$ and hence $f_{y} \in A_{S}(K)$. But $\hat{f}_{y}(\chi)=\chi(y) \hat{f}(\chi)$ for each $\chi \in \hat{K}$ giving $f \in A_{K_{0}}(K)$, i.e. $K_{0}$ is a Helson set. Theorem 4.2 implies $K_{0}$ is finite. Theorem 3.2 of [1] implies $K_{0}=P^{\perp}$ is in one-to-one correspondence with $(\hat{K} / P)^{\wedge}$. Thus $\hat{K} / P$ is finite which implies $\hat{K}$ is countable.

If $H_{x, n}=\{x \in K:|\chi(x)-1|<1 / n\}$ then the sets $H_{x, n}$ form a countable collection of neighborhoods of $e$ with the property

$$
\bigcap_{\substack{x \in \hat{K} \\ n \in \mathbf{Z}^{+}}} H_{\chi, n}=\{e\}
$$


since $\hat{K}$ separates points of $K$. By [13, A.3] we have $K$ is metrizable. Since $S=\operatorname{supp} \mu$ is a closed infinite subset of a compact metric space there is a sequence $\left\{x_{n}\right\}_{n=1}^{\infty}$ of distinct elements of $S$ such that $\lim _{n \rightarrow \infty} x_{n}=x_{0}$. A standard argument can now be used to construct disjoint, nonvoid relatively open subsets $U$ and $V$ of $S$ such that $x_{0} \in U^{-} \cap V^{-}$. Using equation (3), there exists $f \in C(F)$ such that

$$
I_{U} \mu=f \mu \text {, }
$$

i.e. $f(x)=I_{U}(x)$ for $\mu$-almost all $x \in S$. Thus $f(x)=1$ for all $x \in U^{-}$and $f(x)=0$ for all $x \in V^{-}$which contradicts the existence of such a $\mu$.

COROLlary 4.4. Under the hypothesis of Theorem 4.3 every infinite Helson set has m-measure 0 .

Proof. Apply Theorem 4.3 to the measure $\mu$ given by $d \mu=I_{F} d m$. Since all finite sets are Helson sets, there are Helson subsets of nondiscrete compact abelian hypergroups, which have $m$-measure greater than 0 . Indeed, such an example is provided by $F=\{x\}$ in Example 2.6. In fact, Example 2.6 can be used to show that the union of two Helson sets need not be a Helson set. In particular, let $F$ be an infinite Helson set in $G$ and consider $\{x\} \cup F$. Now $\{x\} \cup F$ cannot be a Helson set by Corollary 4.4.

Next we establish a result for Helson sets which is analogous to Theorem 3.2.

THEOREM 4.5. If $K$ is a compact abelian hypergroup which is $n$-fold absolutely continuous, with $Z$ finite and $\hat{K}$ a hypergroup, then $K$ admits no infinite Helson sets.

Proof. As in the proof of Theorem 3.2 we may assume $m(Z)=0$. Let $F$ be a Helson subset of $K$. If $\mu \in M(F)(\mu \neq 0)$ then by [10,6.4] we have $\mu=\mu_{0}+\mu_{h}$ where $\mu_{0} \in M(Z)$ and $\mu_{h} \in M_{h}(K)$. Now by $[10,6.7]$ it follows that $\hat{\mu}_{h} \in c_{0}(\hat{K})$ and hence Theorem 4.3 implies $\operatorname{supp}\left(\mu_{h}\right)$ is finite. $\operatorname{Since} \operatorname{supp}\left(\mu_{0}\right)$ is finite we have every measure on $F$ has finite support, i.e. $F$ is finite.

5. Examples. In this section we consider two examples discussed by Ross in [10, 6.13, 6.17].

EXAMPLE 5.1. Let $G$ be a compact group $\Delta_{p}$ of $p$-adic integers and let $B$ be the group of units in $\Delta_{p}$ acting multiplicatively on $\Delta_{p} . G_{B}$ is a compact abelian hypergroup which is identified with the set $\{0,1,2, \ldots, \infty\}$ and is studied by Dunkl and Ramirez [4]. The hypotheses of Theorems 3.2 and 4.5 apply to $G_{B}$ and hence $G_{B}$ admits no infinite Sidon sets and no infinite Helson sets. This result appears in $[4,10.4]$ for a family of countable compact abelian hypergroups. In fact, Theorems 3.2 and 4.5 of this paper apply equally well to the entire family discussed in [4].

EXAMPLE 5.2. Let $G_{I}$ be the space of conjugacy classes of a compact connected simple Lie group $G$. Results in [8] show that $G_{I}$ satisfies the hypotheses of Theorems 3.2 and 4.5. Thus $G_{I}$ admits no infinite Sidon sets or infinite Helson sets. Ragozin [8] and Rider [9] have shown that $G$ admits no infinite central Sidon sets (and hence $G$ admits no infinite Sidon sets). Using [13, 3.1] it follows that $G$ admitting no infinite central Sidon sets is equivalent to $G_{I}$ admitting no infinite Sidon sets.

The author would like to thank the referee for his useful comments. 


\section{REFERENCES}

1. A. Chilana and A. Kumar, Spectral synthesis in products and quotients of hypergroups, preprint.

2. A. H. Dooley, On lacunary sets for nonabelian groups, J. Austral. Math. Soc. Ser. A 25 (1978), $167-176$.

3. 254-267. , Norms of characters and lacunarity for compact Lie groups, J. Funct. Anal. 32 (1979),

4. C. F. Dunkl and D. E. Ramirez, A family of countable compact $P_{*}$-hypergroups, Trans. Amer. Math. Soc. 202 (1975), 339-356.

5. E. Hewitt and K. A. Ross, Abstract harmonic analysis. I, II, Springer-Verlag, New York, 1963, 1970.

6. R. I. Jewett, Spaces with an abstract convolution of measures, Adv. in Math. 18 (1975), 1-101.

7. J. F. Price, On local central lacunary sets for compact Lie groups, Monatsh. Math. 80 (1975), 201-204.

8. D. L. Ragozin, Central measures on compact simple Lie groups, J. Funct. Anal. 10 (1972), 212-229.

9. D. Rider, Central lacunary sets, Monatsh. Math. 76 (1972), 328-338.

10. K. A. Ross, Centers of hypergroups, Trans. Amer. Math. Soc. 243 (1978), 251-269.

11. W. Rudin, Fourier analysis on groups, Interscience Tracts in Pure and Appl. Math., no. 12, Interscience, New York, 1962.

12. R. C. Vrem, Representations and harmonic analysis of compact hypergroups, Dissertation, University of Oregon, 1978.

13. __ Lacunarity on compact hypergroups, Math. Z. 164 (1978), 93-104.

14. __ Harmonic analysis on compact hypergroups, Pacific J. Math. 85 (1979), 239-251.

Department of Mathematics, Humboldt State University, Arcata, California 95521 